mPTP and was equivalent to the vehicle negative control, indicating no interaction with MPTP in isolated cardiomyocytes. In conclusion, we demonstrate that cardioprotection by MMP inhibition is independent of $\mathrm{CyPD} / \mathrm{mPTP}$ function and can augment the protection seen following iPostC even after prolonged cardiac ischaemia. We believe that this may be through a novel molecular target not implicated in the recognised preconditioning paradigm, and one that may have potential therapeutic implications for clinical practice through attenuating acute reperfusion injury in acute coronary syndromes.

\title{
117 CONDUCTION BLOCK INDUCED BY ACIDOSIS IN HL-1 MOUSE ATRIAL MYOCYTES CAN BE REVERSED BY ADMINISTERING THE GAP JUNCTIONAL COUPLER ROTIGAPTIDE
}

doi:10.1136/heartjnl-2012-301877b.117

S I Al-Aidarous, ${ }^{*}$ C H Roney, F M D Peters, F S Ng, R A Chowdhury, N S Peters. Imperial College London, London, UK

Introduction Gap junctions (GJ) are low resistance intercellular pathways which play a major role in myocardial conduction and their remodelling is a key contributor to arrhythmogenic states. Rotigaptide has been shown to increase GJ coupling after ischaemic stress. However, the effect of rotigaptide in acidotic conditions is not well characterised. We hypothesised that rotigaptide can reverse GJ uncoupling and resultant decreases in conduction velocities (CV) brought about by a low pH in HL-1 mouse atrial myocytes.

Methods A subclone of HL-1 cells were seeded as a drop onto microlectrode arrays and were allowed to form a $2 \mathrm{D}$ monolayer. Baseline recordings were made at a physiological $\mathrm{pH}$ of 7.4 by pacing just above the intrinsic rate at a cycle length of $1000 \mathrm{~ms}$ for $10 \mathrm{~s}$. The same preparations were subsequently incubated in media of pH7.0 for 15 min. Incremental doses of $5 \mathrm{nM}$ of rotigaptide were then added up to a maximum of $100 \mathrm{nM}(\mathrm{n}=8)$. Recordings were taken until no further changes in conduction velocities were seen. Results A reduction of $\mathrm{pH}$ resulted in conduction block in all but one preparation $(8.3 \% \pm 23.6 \%$ of baseline, $\mathrm{p}<0.0001)$ and subse-

A

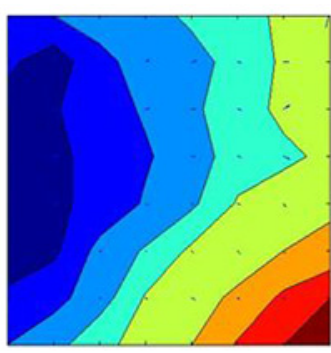

C

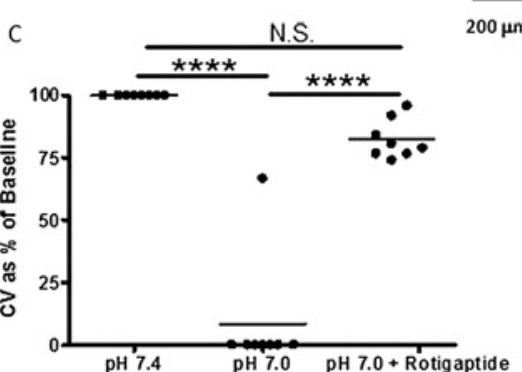

Abstract 117 Figure 1 (A) An activation map from stimulation at baseline $\mathrm{pH}$ 7.4. (B) An activation map from the same array after the cells had been subjected to a low $\mathrm{pH}$ then given rotigaptide. (C) Conduction velocities taken from microelectrode arrays at baseline, $\mathrm{pH}$ 7.0 and $\mathrm{pH} 7.0+$ rotigaptide. ${ }^{* * * *} \mathrm{p}<0.0001$. quent addition of rotigaptide increased the CV $(82.4 \% \pm 7.8 \%$ of baseline, $p<0.0001)$. The CV after addition of rotigaptide was not significantly different to that of the baseline $(p>0.05)$. Activation maps were plotted and the direction of propagation was unchanged ( $\mathrm{p}>0.05)$.

Conclusions Administration of rotigaptide resulted in the reversal of conduction block induced by acidosis without affecting the activation pattern in this atrial cell model. We suggest that acidosis in the absence of ischaemia is a sufficient insult to see an effect with rotigaptide. Therefore rotigaptide may be of use in the reversal of non-ischaemic conduction abnormalities. Further work is required to assess whether rotigaptide can also reverse conduction slowing in whole heart arrhythmogenic states.

\section{FIRST PASS VASODILATOR-STRESS MYOCARDIAL PERFUSION CMR IN MICE ON A CLINICAL WHOLE-BODY 3 TESLA SCANNER: VALIDATION AGAINST MICROSPHERES}

doi:10.1136/heartjnl-2012-301877b.118

R Jogiya, ${ }^{*}$ M Makowski, A Phinikaridou, A Chiribiri, N Zarinabad, S Kozerke, R Botnar, E Nagel, S Plein. Kings College London, London, UK

Background Animal models are important to develop our understanding of the pathophysiology of cardiovascular disease and for the development of new therapies. While coronary autoregulation maintains resting $\mathrm{MBF}$ constant over a wide range of pathological conditions, $\mathrm{MBF}$ reserve during hyperaemic stress is impaired in several common disease processes. First pass contrast-enhanced myocardial perfusion is the standard CMR method for the estimation of $\mathrm{MBF}$ and $\mathrm{MBF}$ reserve in man, but is challenging in rodents because of the constraints related to the high temporal and spatial resolution requirements.

Aim To evaluate first pass vasodilator stress myocardial perfusion CMR of the mouse heart against microspheres as the gold standard for regional organ flow.

Methods Five healthy 6-month old C57BL/6J mice were anaesthetized using $2 \%$ isoflurane. CMR imaging was performed on a clinical 3.0 Tesla scanner (Philips Healthcare, Best, the Netherlands) with a $23 \mathrm{~mm}$ single loop surface coil and a murine monitoring and ECG gating system (SA Instruments, NY, USA). Vasodilator stress was induced using a slow injection of dipyrimadole via a tail vein catheter. Stress perfusion data were acquired with an injection of gadolinium contrast (Gadobutrol $0.5 \mathrm{mmol} / \mathrm{kg}$ ) $30 \mathrm{~s}$ later. The perfusion pulse sequence has been reported1, in summary, it used a saturation recovery gradient echo method with 10 -fold k-space and time domain undersampling with constrained image reconstruction using temporal basis sets ( $k$ - $\mathrm{PCA}$ ) to achieve a spatial resolution of $0.2 \times 0.2 \times 1.5 \mathrm{~mm}^{3}$ and an acquisition window of $43 \mathrm{~ms}$. Following stress perfusion mice were recovered. One week later the mice underwent repeat anaesthesia and stress testing with LV injections of fluorescent microspheres at rest and at stress. Microsphere images were analysed using confocal microscopy.

Results Data were acquired successfully in all five mice. Mean heart rate increased from $480 \pm 27.4 \mathrm{bpm}$ at rest to $503 \pm 41.5 \mathrm{bpm}$ $(p=0.08)$ during vasodilatation. Mean myocardial blood flow at rest by Fermi-function constrained deconvolution in control mice was $3.4 \pm 0.5 \mathrm{ml} / \mathrm{g} / \mathrm{min}$ and increased to $8.9 \pm 3.0 \mathrm{ml} / \mathrm{g} / \mathrm{min}$ during stress (ratio $2.6: 1, \mathrm{p}=0.036$ ). The mean count of microspheres increased from rest to stress by a ratio of 2.7:1 (mean spheres per slice $\mathrm{n}=27 \pm 3.2, \mathrm{n}=74 \pm 18.5, \mathrm{p}=0.0005)$.

Conclusion First-pass myocardial stress perfusion CMR in a mouse model is feasible. Although the quantification of myocardial blood was lower than published values, the trend in myocardial blood flow was consistent with existing literature. Data were acquired on a 3 Tesla scanner using an approach similar to clinical acquisition 
protocols, thus facilitating translation of imaging findings between rodent and human studies to elucidate mechanisms and develop therapies for cardiovascular disease.

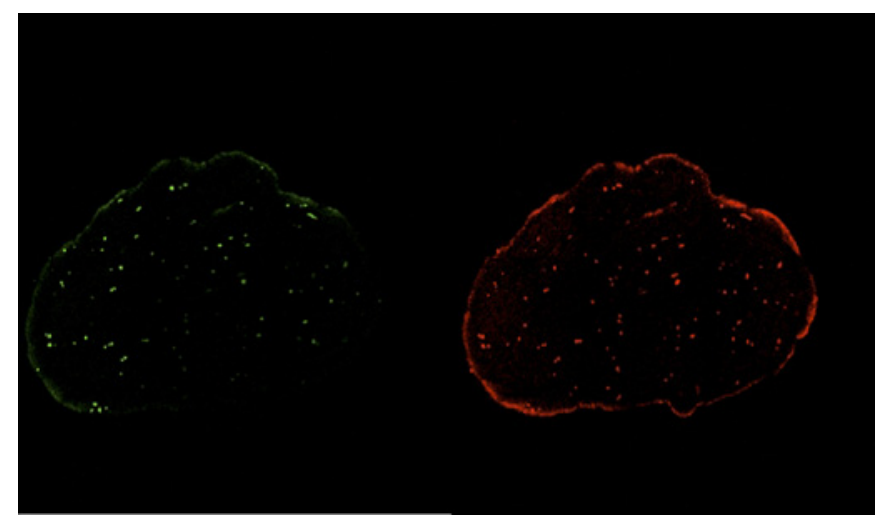

Abstract 118 Figure 1 Fluroescent microsphere injections at rest (green) and stress (red).

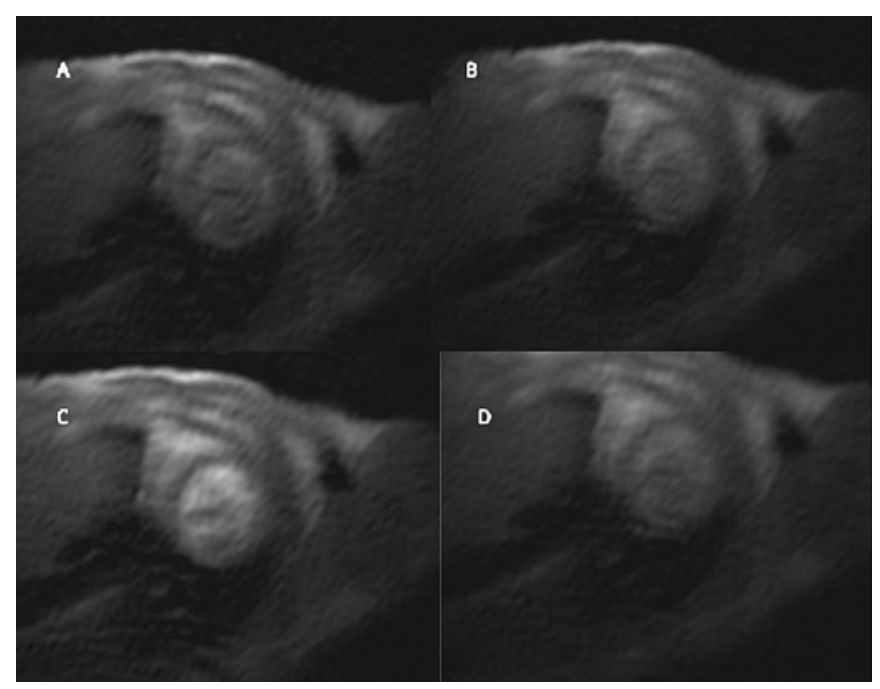

Abstract 118 Figure 2 Murine stress perfusion $(A)=$ rest $(B)=R V$ first pass $(C)=L V$ first pass $(D)=$ myocardial wash in.

\section{SIZE-DEPENDENT RETENTION OF STEM CELLS FOLLOWING INTRACORONARY INJECTION}

doi:10.1136/heartjnl-2012-301877b.119

N G Campbell, ${ }^{*}$ M Kaneko, Y Shintani, C Ikebe, T Narita, V Sawhney, N Tano, S R Coppen, K Yashiro, S Nourshargh, A Mathur, K Suzuki. William Harvey Research Institute, Queen Mary University of London, London, UK

Background Intracoronary (IC) injection of bone marrow mononuclear cells (BMMNCs) is a promising treatment for heart failure. However, therapeutic effects in clinical studies have been small and a principle reason may be related to poor donor cell engraftment. Very early donor cell retention may be an important factor to determine engraftment, but this process is largely unexplored. This study aimed to quantitatively characterise early donor cell retention after IC injection using an original experimental model.

Methods A modified ex-vivo Langendorff perfused rat heart model was developed (Abstract 119 figure 1). Known numbers of rat BMMNCs were injected into the aortic root of the perfused heart ("normal hearts"). The same cell numbers were injected into hearts subjected to $30 \mathrm{~min}$ global ischaemia and $30 \mathrm{~min}$ reperfusion ("I-R hearts"). $8 \times 10^{6}$ rat BM-derived mesenchymal stem cells (MSC), a

\section{(1) Heart perfused with warmed buffer}

(2) Known cell number (a) injected into aortic root via side-port

larger cell type, were also injected into normal hearts. Coronary effluent was continuously collected and cell numbers in the effluent were counted. In this way, the number of cells retained could be quantitatively calculated. The cell size distribution and expression of relevant cell surface markers, both for BMMNC pre-injection and for BMMNC in the coronary effluent, were respectively measured with an automated cell counter and flow cytometry.

Results Median size was 7.0 and $11.0 \mu \mathrm{m}$ for BMMNC and MSC respectively. Most leakage of donor cells into the coronary effluent occurred within $2 \mathrm{~min}$. Calculated retention ratios for the three BMMNC numbers were similar; approximately $20 \%$ in normal hearts increasing to 30\% after I-R (Abstract 119 table 1). The retention ratio for MSC injection was much higher. Surface markers of BMMNCs were unchanged between pre-injection and coronary effluent cells. Instead, it was found larger BMMNC were more frequently retained in normal hearts (Abstract 119 table 2). This retention pattern was magnified in I-R hearts: larger BMMNC were retained 6 times more efficiently than smaller cells. Histological studies using $\mathrm{PKH}$-stained BMMNCs demonstrated that all BMMNC were observed within the lumen of small vessels and none

Abstract 119 Table 1 Cell retention up to $5 \mathrm{~min}$

\begin{tabular}{llll}
\hline Cell number injected & $\mathbf{1 \times 1 0 ^ { \mathbf { 6 } }}$ & $\mathbf{8} \times \mathbf{1 0}^{\mathbf{6}}$ & $\mathbf{4 0 \times 1 0 ^ { \mathbf { 6 } }}$ \\
\hline BMMNC-Normal Heart (\%) & $20.0 \pm 3.5$ & $21.1 \pm 2.2$ & $23.5 \pm 1.9$ \\
BMMNC-I-R Heart (\%) & $31.7 \pm 2.8^{*}$ & $30.9 \pm 1.2^{*}$ & $29.4 \pm 1.7^{*}$ \\
MSC-Normal Heart (\%) & & $77.8 \pm 4.1^{*}$ &
\end{tabular}

Mean \pm SEM, $n \geq 4$ each group.

${ }^{*} p<0.05$ vs same cell number BMMNC-Normal Heart.

Abstract 119 Table 2 BMMNC-size dependent retention

\begin{tabular}{|c|c|c|c|}
\hline Cell size & $5-6 \mu \mathrm{m}$ & $7 \mu \mathrm{m}$ & $\geq 8 \mu \mathrm{m}$ \\
\hline Initial BMMNC fraction (\%) & $42.0 \pm 3.6$ & $28.2 \pm 0.8$ & $29.8 \pm 3.0$ \\
\hline \multicolumn{4}{|l|}{ Retention up to $5 \mathrm{~min}(\%)$} \\
\hline Normal Heart & $14.5 \pm 7.3$ & $30.3 \pm 4.2$ & $39.8 \pm 6.1^{*}$ \\
\hline I-R Heart & $9.5 \pm 2.6$ & $40.5 \pm 2.8^{*}$ & $58.5 \pm 2.4^{* * *}$ \\
\hline
\end{tabular}

Mean $\pm S E M, n \geq 5$ each group.

${ }^{*} \mathrm{p}<0.05$ vs $5-6 \mu \mathrm{m}$, ${ }^{* *} \mathrm{p}<0.05$ vs $7 \mu \mathrm{m}$ 